\title{
The prognostic value of quantitative analysis of CCL5 and collagen IV in luminal B (HER2-) subtype breast cancer by quantum-dot-based molecular imaging
}

This article was published in the following Dove Press journal: International Journal of Nanomedicine

\section{Yong-yun Zhu' \\ Chuang Chen ${ }^{2}$ \\ Juan-Juan $\mathrm{Li}^{2}$ \\ Sheng-Rong Sun ${ }^{2}$ \\ 'Department of Thyroid and Breast Surgery, Wuhu Second People's Hospital, Wuhu, Anhui 24l00, People's Republic of China; ${ }^{2}$ Department of Breast and Thyroid Surgery, Renmin Hospital of Wuhan University, Wuhan, Hubei 430060, People's Republic of China}

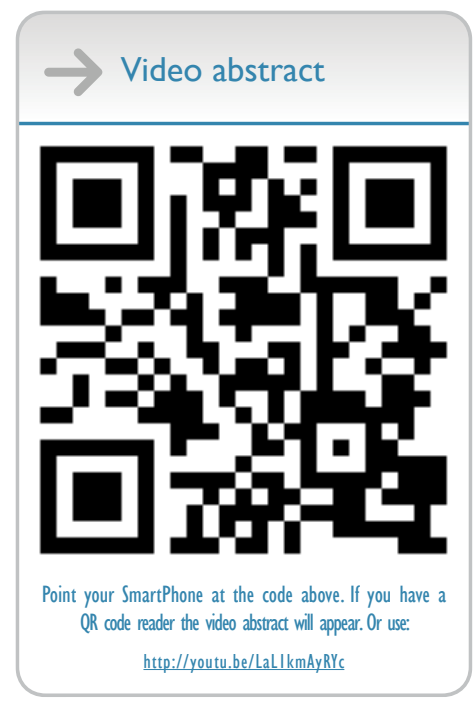

Correspondence: Chuang Chen Department of Breast and Thyroid Surgery, Renmin Hospital of Wuhan University, No 238, Jiefang Road, Wuchang District, Wuhan, Hubei 430060, People's Republic of China Tel +86 I3476064785 Email18055316143@163.com
Objective: Breast cancer is the most common malignancy and one of the main causes of death in women. Luminal B (HER2-) breast cancer subtype has been proposed since the $2011 \mathrm{St}$ Gallon consensus. The hormone receptor status in this type of breast cancer is positive; thus, endocrine therapy was performed in all cases, but the treatment was not satisfactory, and a significant number of cases received very little benefit from chemotherapy. Furthermore, there is no effective treatment target for this subtype. Luminal B (HER2-) breast cancer subtype has been proposed since the $2011 \mathrm{St}$ Gallon consensus. Therefore, the study of the key molecules in the microenvironment of breast cancer can help to reveal the biological characteristics.

Patients and methods: Luminal B (HER2-) breast cancer is a subtype with higher heterogeneity and poorer prognosis than luminal A. It is known that the development of cancer cells is an active process, and this process needs microenvironment cytokines, including chemokine (C-C motif) ligand 5 (CCL5) and collagen IV. Therefore, CCL5 and collagen IV were imaged and detected by quantum dot, and the CCL5/collagen IV ratio was calculated to investigate the prognostic value of the CCL5/collagen IV ratio in luminal B (HER2-).

Results: Quantitative determination showed a statistically significant negative correlation between CCL5 and collagen IV. The 5-year disease-free survival (5-DFS) of the high and low CCL5/collagen IV ratio subgroups was significantly different. The CCL5/collagen IV ratio had a greater prognostic value for 5-DFS. The CCL5/collagen IV ratio was an independent prognostic indicator.

Conclusion: Our findings revealed the effective integration of tumor CCL5 and collagen IV, and a new method for predicting the prognosis of luminal B (HER2-) has been developed. Keywords: quantum dot, luminal B, HER2-, chemokine, C-C motif, ligand 5, collagen IV

\section{Introduction}

Breast cancer is the most common malignancy and one of the main causes of death in women. ${ }^{1}$ Luminal B (HER2-) subtype breast cancer is defined as (estrogen receptor $[\mathrm{ER}]+)$, (progestogen receptor $[\mathrm{PR}]+;<20 \%$ ), HER2- and Ki-67 $\geq 14 \%$ disease. $^{2}$ According to the St Gallen 2015 definitions, the proportion of luminal (HER2-) cancer is $71 \%$ and luminal B-like (HER2+) is $6 \%,{ }^{3}$ and race/ethnicity and socioeconomic status are independent in contributing to disease among luminal A tumors, but not found in women with luminal B (HR+/HER2+) disease. ${ }^{4}$ The clinicopathological features of luminal B breast cancer are similar to those of luminal A breast cancer, but luminal $\mathrm{B}$ cancer has a higher heterogeneity and poorer prognosis than luminal $\mathrm{A},{ }^{5,6}$ and there is a higher proportion of local recurrence and single bone metastasis in patients with 
luminal B breast cancer than in patients in the nonluminal groups. ${ }^{7}$ The hormone receptor status in this type of breast cancer is positive; thus, endocrine therapy was performed in all cases, but the treatment was not satisfactory, ${ }^{8}$ and a significant number of cases received very little benefit from chemotherapy. Furthermore, there is no effective treatment target for this subtype. Luminal B (HER2-) breast cancer subtype has been proposed since the 2011 St Gallon consensus. This subtype of breast cancer has been extensively studied. ${ }^{9-16}$ It is known that the microenvironment is closely related to the occurrence and development of tumor. ${ }^{17,18}$ Therefore, the study of the key molecules in the microenvironment of breast cancer can help to reveal the biological characteristics. Imaging technology of nanoparticle quantum dot (QD)-labeled molecular probes is a new technology that is based on nanomaterials and immunofluorescence detection. It has characteristics such as good histocompatibility, fluorescence signal stabilization and multimolecule coaxial imaging. We have already applied this technology to study some key molecules in breast cancer such as HER2, ER, EGFR and Ki-6 $7^{19-22}$ and some key molecules in microenvironment such as collagen IV, ${ }^{23}$ and the co-imaging of the key molecules between cancer cells and the tumor microenvironment (TM) was also successfully performed. ${ }^{24}$ But how the key molecules participate in the occurrence and development of tumor through the microenvironment needs further study.

CCL5 is one of the key breast cancer molecules which is closely related to invasion and metastasis of breast cancer. ${ }^{25,26}$ Exploring the dynamic relationship between CCL5 and breast cancer microenvironment would help to reveal the mechanism of tumor invasion and metastasis. Invasion and metastasis of breast cancer are a multistep complex process, which include laminine bound to extracellular matrix (ECM), released protein hydrolase, degraded ECM, passed through basement membrane (BM) transfer and so on. ECM is the main tissue barrier of tumor cell infiltration, mainly composed of collagen IV and others, which can prevent the invasion and metastasis of tumor cells. Exploring the dynamic relationship between CCL5 and breast cancer microenvironment is helpful to reveal the process of tumor invasion and metastasis.

Traditional immunohistochemical (IHC) technique can only perform semiquantitative detection of a single tumor key molecule; however, the imaging technique of nano-QDlabeled molecular probes can realize multimolecule coaxial imaging. ${ }^{19}$ Some scholars have applied this technology to study the key molecules of breast cancer. ${ }^{19,27,28}$ We have successfully applied this technique in the previous study. ${ }^{20-24,29,30}$ Based on earlier studies, we used luminal B (HER2-) breast cancer as the study sample, performing molecular co-imaging of CCL5 and collagen IV, to investigate the mechanism of invasion and metastasis of luminal B (HER2-) breast cancer. Meanwhile, we further constructed the quantitative evaluation system of CCL5 and collagen IV by image acquisition system to find out new prognostic markers for luminal B (HER2-) breast cancer.

\section{Patients and methods}

\section{Patients and specimens}

Formalin-fixed, paraffin-embedded specimens from 109 patients with luminal B HER2- invasive breast cancer were collected from the Second People's Hospital of Wuhu, China, from January 2009 to January 2012. All patients are women, aged from 24 to 79 years, with a median age of 49 years. There were 44 cases with lymph node metastases and 65 without lymph node metastases. Major pathological parameters, including age, tumor size, lymph node status and histological grade as determined by conventional IHC, were available. All patients had undergone six to eight cycles of anthracycline- and docetaxel-based chemotherapy after breast cancer surgery; patients who had $>3$ metastatic lymph nodes had undergone radiotherapy after chemotherapy; and all patients had undergone endocrinotherapy after chemotherapy (tamoxifen was used pre menopause, and letrozole was used after menopause). The end point of the follow-up study was recurrence or distant metastasis. The study protocol was approved by the medical ethics committee of the Second People's Hospital of Wuhu. All the patients whose tissue samples were used had provided written informed consent. The characteristics of patients are summarized in Table 1.

Table I Correlation between CCL5/collagen IV ratio and clinicopathological features of breast cancer

\begin{tabular}{|c|c|c|c|}
\hline \multirow[t]{2}{*}{ Items } & \multirow[t]{2}{*}{ Number (\%) } & \multicolumn{2}{|c|}{ CCL5/collagen IV } \\
\hline & & Ratio ( $\bar{x} \pm s)$ & $P$-value \\
\hline Age (years) & & & 0.179 \\
\hline $24<$ age $\leq 40$ & $16(14.68)$ & $0.53 \pm 0.64$ & \\
\hline $40<$ age $\leq 60$ & $72(66.05)$ & $0.54 \pm 0.50$ & \\
\hline$>60$ & $21(19.27)$ & $0.87 \pm 0.34$ & \\
\hline Tumor size $(\mathrm{cm})$ & & & 0.001 \\
\hline $\mathrm{T0}(\mathrm{T} \leq 2)$ & $4 I(37.6 I)$ & $0.27 \pm 0.6 \mathrm{I}$ & \\
\hline $\mathrm{TI}(\mathrm{T}>2)$ & $68(62.39)$ & $0.54 \pm 0.41$ & \\
\hline Lymph node status & & & 0.017 \\
\hline Negative & $65(59.63)$ & $0.34 \pm 0.38$ & \\
\hline Positive & $44(40.37)$ & $0.59 \pm 0.62$ & \\
\hline Tumor grade & & & 0.790 \\
\hline Grade I & $26(23.85)$ & $0.38 \pm 0.46$ & \\
\hline Grade 2 & $58(53.21)$ & $0.47 \pm 0.57$ & \\
\hline Grade 3 & $25(22.94)$ & $0.44 \pm 0.50$ & \\
\hline
\end{tabular}

Abbreviation: CCL5, chemokine (C-C motif) ligand 5. 


\section{CCL5 testing}

Four-micrometer-thickness tissue sections were heated for $1.5 \mathrm{~h}$ at $60^{\circ} \mathrm{C}$ and then dewaxed three times in xylene solution (each time for $6 \mathrm{~min}$ ). Tissue sections of dewaxing were hydrated by alcohol of descending concentration ( $100 \%$ ethanol $\rightarrow 95 \%$ ethanol $\rightarrow 85 \%$ ethanol $\rightarrow 75 \%$ ethanol $\left.\rightarrow \mathrm{H}_{2} \mathrm{O} \rightarrow \mathrm{ddH}_{2} \mathrm{O}\right)$. Tissue sections of hydration were pretreated in citric acid buffer for antigen retrieval $\left(0.01 \mathrm{M} / \mathrm{L}, \mathrm{pH} 6.0 ; 100^{\circ} \mathrm{C}\right.$ citric acid buffer $\rightarrow \mathrm{H}_{2} \mathrm{O} \rightarrow \mathrm{ddH}_{2} \mathrm{O} \rightarrow$ Tris-buffered saline). Tissue sections of antigen retrieval were added to the blocking buffer $\left(3 \% \mathrm{H}_{2} \mathrm{O}_{2}\right)$, then incubated at $37^{\circ} \mathrm{C}$ for $10 \mathrm{~min}$ and, finally, rinsed three times by Tris-buffered saline (every time for $3 \mathrm{~min}$ ). After removing the blocking buffer, slides were added to mouse antihuman CCL5 (1:100; 53405.111; Abcam, Cambridge, UK) and then incubated at $37^{\circ} \mathrm{C}$ for $2 \mathrm{~h}$. The slides were rinsed with mixed solution of Tris-buffered saline and Tween-20 (2,000:1) and then added to the blocking buffer after removing the rinsed solution, followed by incubating at $37^{\circ} \mathrm{C}$ for $30 \mathrm{~min}$. The slides were added to biotin-labeled sheep anti-rabbit immunoglobulin G (IgG) (dilution 1/100; Vector Laboratories, Inc., Burlingame, CA, USA) after removing the blocking buffer and then incubated at $37^{\circ} \mathrm{C}$ for $1 \mathrm{~h}$. The slides were added to quantum dots conjugated streptavidin (QDs-SA) probe ( $\lambda=605 \mathrm{~nm}$; Jiayuan Quantum Dots, Wuhan, China) after blocking and then incubated at $37^{\circ} \mathrm{C}$ for $30 \mathrm{~min}$. The slides were sealed by mixed solution of Tris-buffered saline, and there was no fluorescence glycerol (1:9) after rinsing; the slides were then observed using fluorescence microscope (Leica DM 4000B; Leica Microsystems, Wetzlar, Germany) in Blu-ray ( $\lambda=405-565 \mathrm{~nm}$ ), finally, stored at $4^{\circ} \mathrm{C}$ by avoiding light.

\section{CCL5 and collagen IV testing}

The main steps for the imaging of CCL5 and collagen IV are as follows: heating $\left(60^{\circ} \mathrm{C}, 1.5 \mathrm{~h}\right) \rightarrow$ dewaxing (xylene solution) $\rightarrow$ hydration (alcohol-gradient hydration) $\rightarrow$ antigen retrieval (citric acid buffer pretreatment) $\rightarrow$ blocking (Trisbuffered saline) $\rightarrow$ adding the first antibody (mouse antihuman CCL5; rabbit antihuman collagen IV, 1:200, EPR17072; Abcam) $\rightarrow$ rinsing (mixed solution of Tris-buffered saline and Tween-20, 200:1) $\rightarrow$ blocking (Tris-buffered saline) $\rightarrow$ adding the second antibody (biotinylated IgG) $\rightarrow$ rinsing (mixed solution of Tris-buffered saline and Tween-20, 200:1) $\rightarrow$ blocking (Tris-buffered saline) $\rightarrow$ adding QDs-SA (605-QDs-SA, 525 QDs-SA) $\rightarrow$ rinsing (mixed solution of Tris-buffered saline and Tween-20, 200:1) $\rightarrow$ sealing (mixed solution of Tris-buffered saline and no fluorescence glycerol, 1:9) $\rightarrow$ observation (the fluorescence microscope, $\lambda=330-385 \mathrm{~nm}$ ). In addition to the three experimental steps, such as adding the first antibody, adding QDs-SA and observation, the other steps were the same as the CCL5 experiment. In the process of adding the first antibody, mouse antihuman CCL5 (1:100) and rabbit antihuman collagen IV (1:200) were blending (1:1). In the process of adding QDs-SA, 605-QDs-SA and 525-QDs-SA were blending (1:1). The experimental results were observed by fluorescence microscope under ultraviolet (UV) excitation: 605-QDs-SA (inorganic components: $\mathrm{ZnCdSe} / \mathrm{ZnS}$; surface ligands: oleic acid; size: $7.5 \pm 0.5 \mathrm{~nm}$; emission: $604 \mathrm{~nm}$; full width at half maximum: $29 \mathrm{~nm}$; quantum yield: 92\%; MaiXin-Bio, Fuzhou, China) and 525-QDs-SA (inorganic components: $\mathrm{ZnCdSe/ZnS;} \mathrm{surface} \mathrm{ligands:} \mathrm{oleic} \mathrm{acid;} \mathrm{size:}$ $5.5 \pm 0.5 \mathrm{~nm}$; emission: $521 \mathrm{~nm}$; full width at half maximum: $34 \mathrm{~nm}$; quantum yield: 75\%; MaiXin-Bio; Figure 1).

\section{Image acquisition and analysis}

The molecular imaging of QDs was acquired by Olympus DP72 image acquisition system (Olympus Corporation, Tokyo, Japan). The QD fluorescence signal information on CCL5 and collagen IV was analyzed as mentioned earlier. The distribution and expression intensity of QDs were quantified by spectral stripping technique, and the QD score (QDS) of each sample was obtained.

\section{Statistical analyses}

The relationship between the CCL5/collagen IV ratio and clinical characteristics was assessed by the Mann-Whitney U test or the Kruskal-Wallis H test, and Spearman correlation analysis was adopted. The optimal boundary value point was defined by the curve analysis of the subject (receiver operating characteristic curve [ROC]). The single-factor survival analysis was carried out by Kaplan-Meier method. The log-rank examination and evaluation group was used to evaluate the difference. Cox proportional risk model was used to evaluate the independent prognostic factors of breast cancer. Statistical analyses were performed by SPSS 19.0 software (IBM Corporation, Armonk, NY, USA), and twotailed $P<0.05$ was considered as statistically significant.

\section{Results \\ CCL5 and collagen IV detection and quantification in luminal B (HER2-) tissue}

We found that CCL5 fluorescence of orange red QDs with specific distribution in the cytoplasm of cancer cell (Figure 2A) using QD-F(ab)2 probes excited by a single UV excitation light source (UV 330-385 nm), software separation of CCL5 QD fluorescence (Figure 2B), CCL5 and collagen IV coimaging, CCL5 fluorescence imaging is orange red in the cytoplasm, collagen IV fluorescence imaging is green in the BM (Figure 2C), software separation of CCL5 and collagen IV 


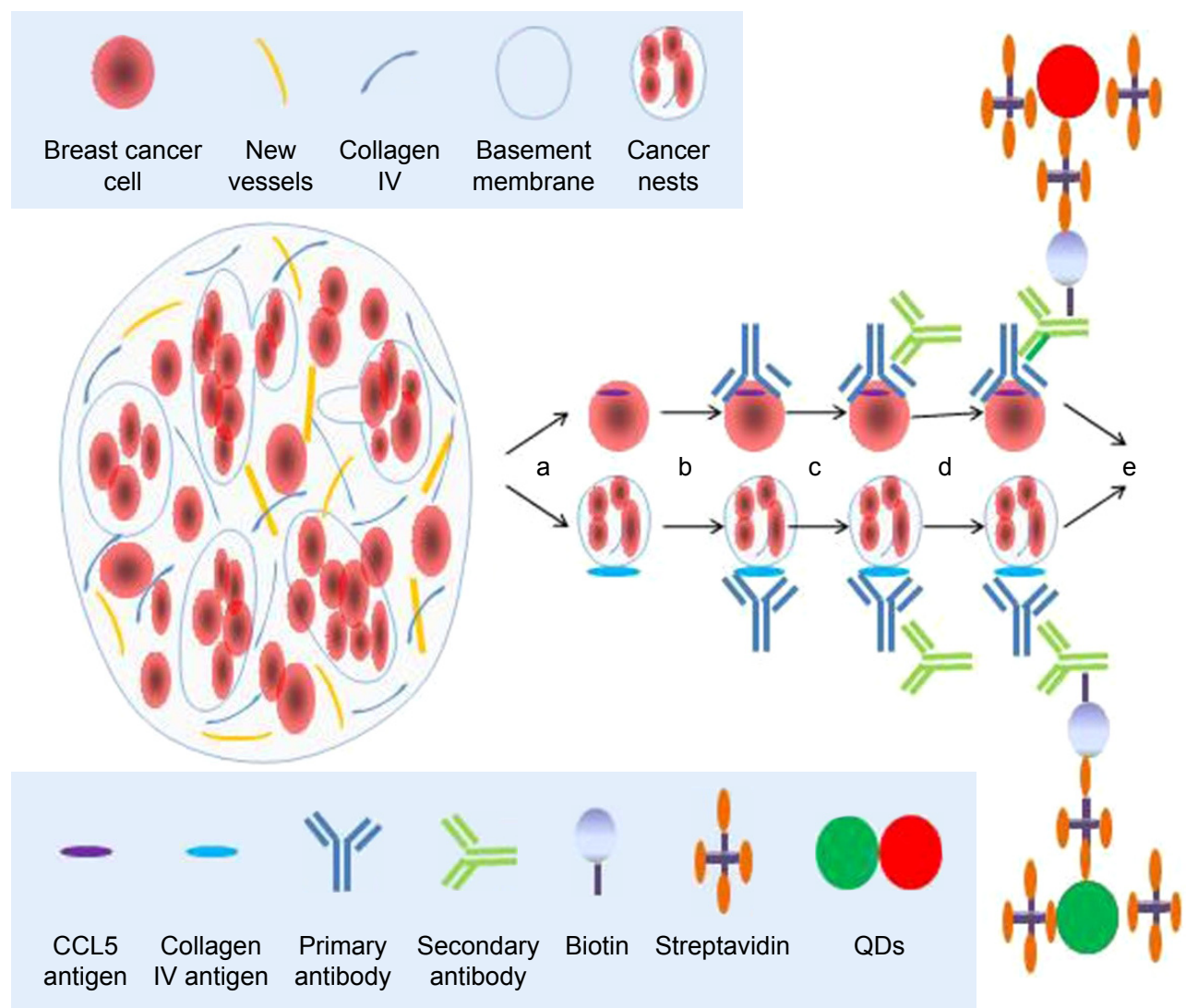

Figure I CCL5 and collagen IV testing.

Notes: CCL5 expressed in the cytoplasm of cancer cell and collagen IV expressed in the BM were imaged and detected simultaneously and quantitatively by QD-based system. a, heating, dewaxing, hydration; b, antigen retrieval, blocking, adding the first antibody; c, rinsing, blocking, adding the second antibody; d, rinsing, blocking, adding QDs-SA; e, rinsing, sealing, observation.

Abbreviations: BM, basement membrane; CCL5, chemokine (C-C motif) ligand 5; QD, quantum dot.

QD fluorescence (Figure 2D), spectrally isolated CCL5 single expression signals (Figure 2E), spectrally isolated collagen IV single expression signals (Figure 2F). In 109 patients, there was statistically significant negative association between CCL5 and collagen IV (Spearman's $\rho, P=0.001, r=-0.315$ ).

\section{Relationship between CCL5/collagen IV ratio and clinicopathological traits}

The relationship between CCL5/collagen IV ratio and clinicopathological traits, including age, tumor size, lymph node status and tumor grade, was evaluated. The CCL5/collagen IV ratio was significantly correlated with tumor size and lymph node status. There was no significant correlation with age and tumor grade (Table 1).

\section{CCL5 and CCL5/collagen IV ratio expression according to 5-year disease-free survival (5-DFS)}

ROC analysis of the CCL5 and CCL5/collagen IV ratio expression according to 5-DFS indicated that they could predict 5-DFS (Figure 3). According to the optimal sensitivity and specificity of the ROC curve that was based on the 5-DFS status, we chose 54.5 and 0.79 as the cutoffs for the CCL5 and CCL5/collagen IV ratio expression, respectively, where the CCL5 expression had $75.9 \%$ sensitivity and $60.0 \%$ (Figure $3 \mathrm{~A})$ specificity and the CCL5/collagen IV ratio had $75.9 \%$ sensitivity and $67.5 \%$ specificity (Figure $3 \mathrm{~B}$ ). The CCL5/collagen IV ratio had higher specificity and a larger area under the curve than CCL5 expression, suggesting that the ratio has better prognostic value for 5-DFS than CCL5 expression.

\section{CCL5 and CCL5/collagen IV ratio expression and 5-DFS}

In this study, the 5-DFS rate was 73.4\% (80/109) with 19 local recurrences and 10 distant recurrences. We classified the patients as high and low CCL5 expression subgroups that were based on the CCL5 expression cutoff value of 54.5. The 5-DFS rate was $87.3 \%$ for the patients who had low CCL5 ( $\mathrm{n}=55)$ and $59.3 \%$ for the patients who had high CCL5 ( $\mathrm{n}=54)$, the difference was significant $(P=0.001$, 

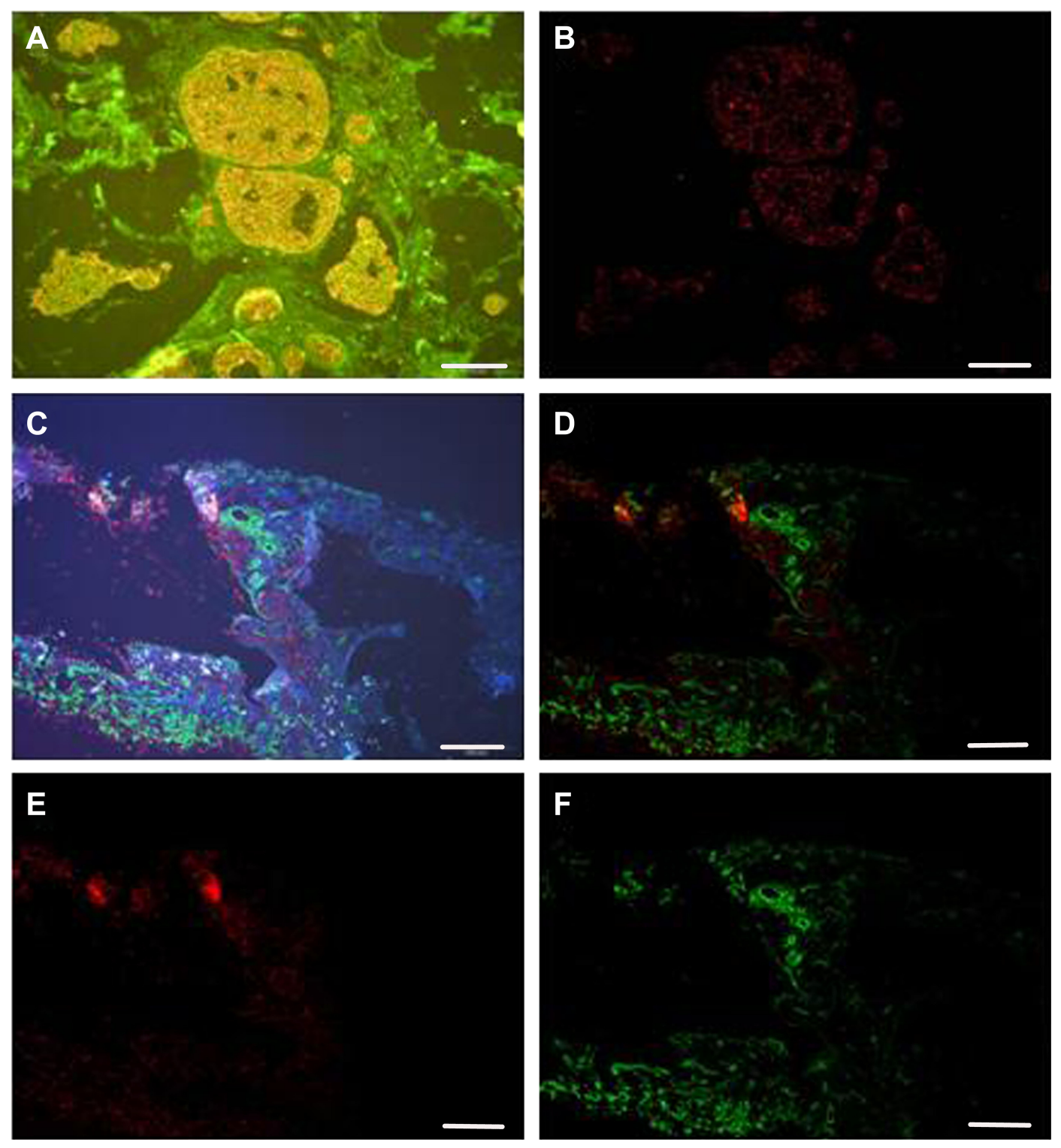

Figure 2 CCL5 and collagen IV detection and quantification in luminal B (HER2-) tissue.

Notes: (A) CCL5 fluorescence of orange red QDs with specific distribution in the cytoplasm of cancer cell. (B) Software separation of CCL5 QD fluorescence. (C) CCL5 (red) on the cytoplasm of cancer cell and collagen IV (green) in the ECM were visible at high resolution against the clear discernable background. (D) Software separation of CCL5 and collagen IV QD fluorescence. (E) Spectrally isolated CCL5 single expression signals. (F) Spectrally isolated collagen IV single expression signals. Scale bar=I00 $\mu$ m. Abbreviations: $\mathrm{CCL} 5$, chemokine ( $\mathrm{C}-\mathrm{C}$ motif) ligand 5; $\mathrm{ECM}$, extracellular matrix; $\mathrm{QD}$, quantum dot.

log-rank test; Figure 4A). Based on the CCL5/collagen IV ratio cutoff value of 0.79 , the 109 patients were classified as the high and low CCL5/collagen IV ratio subgroups. The 5 -DFS rate was $88.5 \%$ for the patients who had low CCL5/ collagen IV ratio $(n=61)$, and $54.2 \%$ for the patients who had high CCL5/collagen IV ratio $(n=48)$. The 5-DFS of these two groups was significantly different $(P<0.001$, log-rank test; Figure 4B).

Multivariable analyses revealed that the CCL5/collagen IV ratio (cutoff 0.79) was predictive in breast cancer and was an independent prognostic indicator (Table 2).

\section{Discussion}

It is known that the TM, serving pivotal role in tumor development, is an important factor in identifying recurrence and promoting metastasis. ${ }^{17,31,32}$ Chemokines can regulate the TM-driven mammary carcinomas. ${ }^{33}$ CCL5 is a novel therapeutic target for estrogen-dependent breast cancer ${ }^{34}$ and is essential for tamoxifen resistance in human breast cancer cells. ${ }^{35} \mathrm{~B}$ subtype breast cancer is a relatively poor prognosis for Luminal A subtype breast cancer, especially luminal B (HER2-) has no effective therapeutic target. This study focused on luminal B (HER2-) and CCL5 to explore 

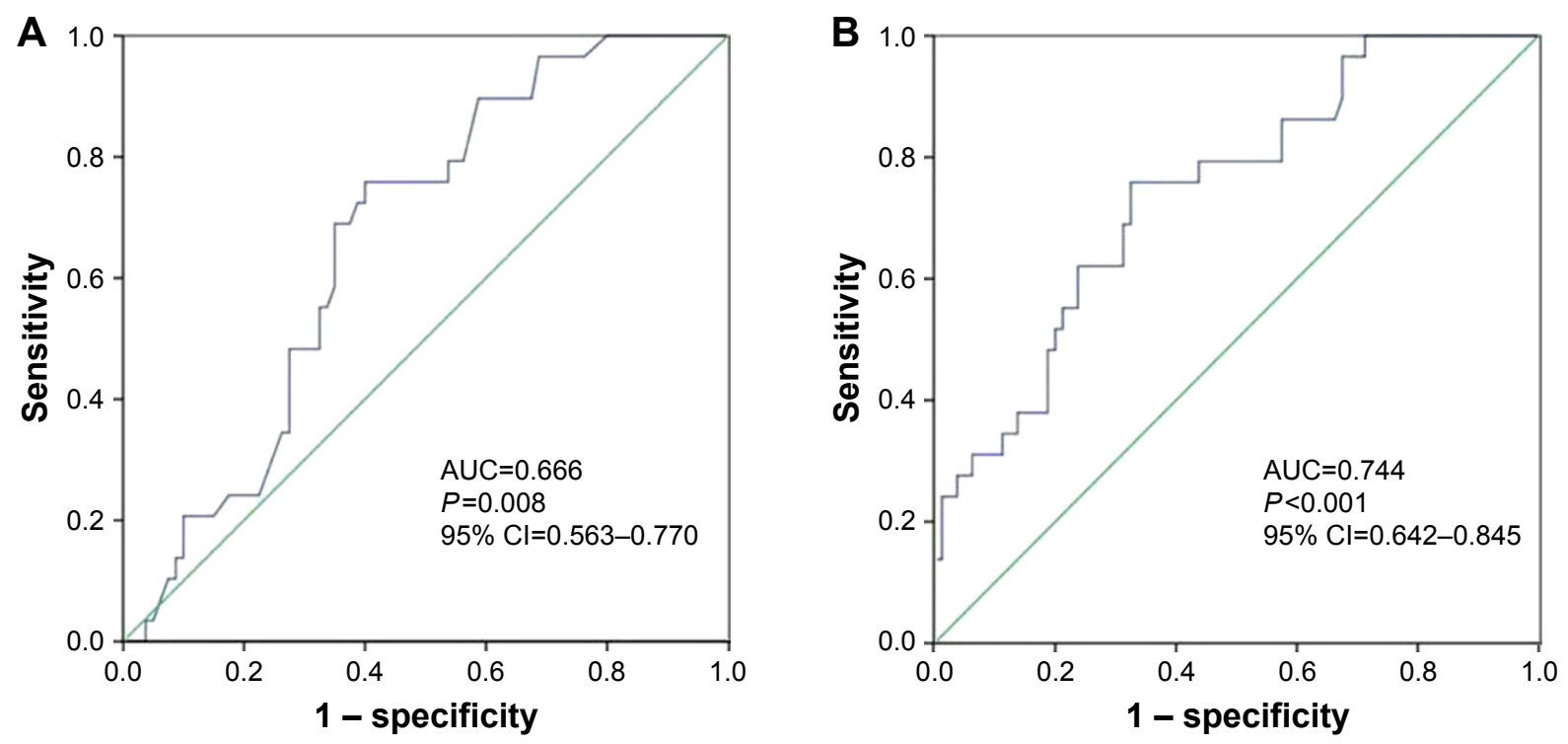

Figure 3 ROC analysis of the CCL5 and CCL5/collagen IV ratio expression according to 5-DFS.

Notes: $(A) C C L 5$ ROC (sensitivity $=0.759$, specificity $=0.60, P=0.008$, cutoff $=54.5, A U C=0.666,95 \%$ Cl=0.563-0.770). (B) $C C L 5 /$ collagen IV ratio $R O C$ (sensitivity=0.759, specificity $=0.675, P<0.00$ I, cutoff $=0.79, \mathrm{AUC}=0.744,95 \% \mathrm{Cl}=0.642-0.845)$.

Abbreviations: AUC, area under the curve; CCL5, chemokine (C-C motif) ligand 5; 5-DFS, 5-year disease-free survival; ROC, receiver operating characteristic.

possible therapeutic targets for this type of breast cancer. The existing CCL5 detection method of breast cancer is mainly semiquantitative IHC, ${ }^{36}$ but we have successfully visualized and quantitatively detected CCL5 by using QD technology. Subsequently, the follow-up results showed that the prognosis of high CCL5 expression group was significantly worse than that of low expression group, indicating that high CCL5 expression is related to tumor infiltration and metastasis and poor prognosis; it was consistent with many studies in recent years. ${ }^{37,38}$ Collagen IV is an important component of ECM in
TM. Therefore, we focused on CCL5 and collagen IV at the same time. QD technology is applied to implement the CCL5 and collagen IV co-imaging; this makes it possible to monitor two indicators of the same samples of breast cancer microenvironment at the same time to become a reality, so that we can observe the dynamic changes between the two indicators. In the study of luminal B (HER2-) breast cancer, we found that there was statistically significant negative association between CCL5 and collagen IV. A number of studies have confirmed that CCL5 is closely related to tumor infiltration
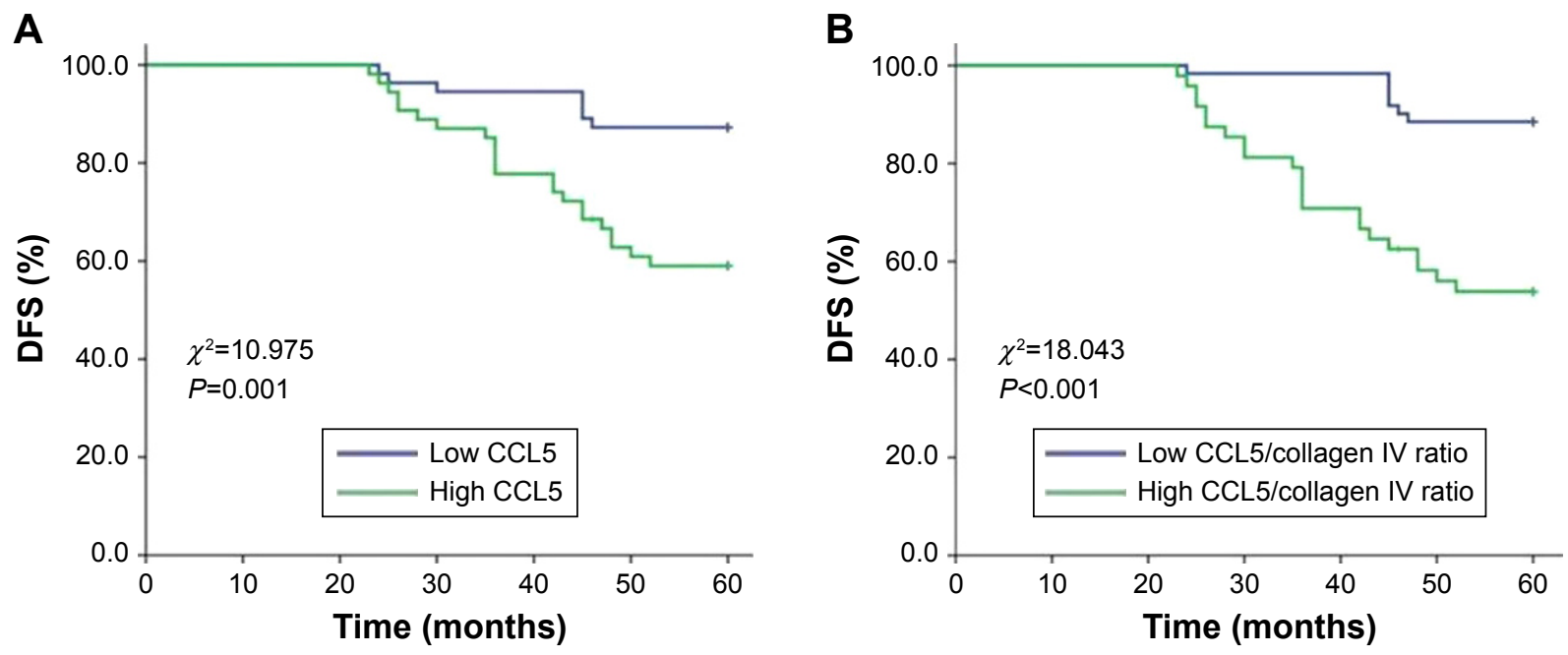

Figure 4 CCL5 and CCL5/collagen IV ratio and 5-DFS.

Notes: (A) CCL5 and 5-DFS, the cutoff value was 54.5; the 5-DFS of these two groups was significantly different ( $P=0.00 \mathrm{I}$, log-rank test). (B) CCL5/collagen IV ratios and 5 -DFS, the cutoff value was 0.79 ; the 5 -DFS of these two groups was significantly different $(P<0.00 \mathrm{I}, \log$-rank test).

Abbreviations: CCL5, chemokine (C-C motif) ligand 5; 5-DFS, 5-year disease-free survival. 
Table 2 Multivariate analysis of 5-DFS in 109 patients with breast cancer

\begin{tabular}{llll}
\hline Items & Wald & RR $(95 \%$ CI) & $P$-value \\
\hline Age & 0.646 & $0.766(0.399-I .468)$ & 0.422 \\
Tumor grade & 0.028 & $0.95 I(0.53 I-1.704)$ & $0.95 I$ \\
Lymph node status & 9.240 & $3.706(1.593-8.624)$ & 0.002 \\
Tumor size & 2.506 & $2.407(0.8 I I-7.138)$ & 0.113 \\
CCL5/collagen IV ratio & 8.153 & $3.548(1.488-8.464)$ & 0.004 \\
\hline
\end{tabular}

Note: aCutoff, 0.79 .

Abbreviations: $\mathrm{CCL}$, chemokine (C-C motif) ligand 5; 5-DFS, 5-year disease-free survival; RR, relative risk.

and metastasis, ${ }^{39-42}$ whereas this study further suggests that CCL5 promotes metastasis associated with collagen IV.

The accurate classification of tumor is the premise of individualized treatment of tumor. In recent years, the further classification of luminal breast cancer has attracted much attention. HR+/HER2- tumors were divided into two groups, which were based on the difference between luminal A/B signature (protein expression of ER, AR, Bcl-2, Bim [BCL2L11], GATA3 and INPP4b).$^{43}$ Luminal B breast tumor is more common, but this type has not been investigated thoroughly, because it has aggressive clinical and biological features and constitutes the most heterogeneous molecular subtype, both clinically and molecularly. The luminal B group can be divided into subtypes according to the expression of Ki-67 (cutoff at 25\%). ${ }^{44}$ Unfortunately, the classification of luminal B subtype breast cancer remains still imprecise by immunohistochemistry, but it is important to define a classification scheme capable of segregating luminal tumors into clinically meaningful subgroups that may be used clinically to guide patient management. ${ }^{6}$ According to the St Gallen 2015 guidelines, the proportion of BCs classified as luminal B (HER2-) is highly influenced by the Ki67-LI assessment method. But there is no standardized assessment method. $^{3}$ APOA1, GELS, HS90B, EF1A1, NHRF1 and PRDX3 can identify the type of luminal B breast cancer. ${ }^{45}$ Attentions are focused on the tumor biomarkers, but the growth of tumor requires microenvironment. ${ }^{46-49}$ In this study on the basis of synchronous detection of CCL5 and collagen $\mathrm{IV}$, to evaluate further the prognostic value of CCL5/collagen IV ratio, we found that the prognosis of the high CCL5/collagen IV ratio group was significantly worse than the low CCL5/collagen IV ratio group, and the CCL5/collagen IV ratio prediction value was superior to the predicted value of CCL5. Multivariable analyses revealed that the CCL5/ collagen IV ratio was an independent predictor. Therefore, it offered more options at the time of treatment; we need to pay attention on the tumor itself and the microenvironment of the tumor as well. Although we explored a new belief for the treatment of patients with luminal B (HER2-) breast cancer, we need to combine more information than study the relationship between them to identify different subtypes through QDbased in situ simultaneous imaging and quantitative analysis to guide individualized and effective treatment.

We used QD-based imaging and computer-aided quantitative analysis to image and quantitatively analyze the key molecules CCL5 and collagen IV successfully in luminal B (HER2-). Our findings demonstrated the QD-based multiplex multispectral technique can be highly sensitive, colocated molecular imaging and quantitative analysis. This technique may help us to assess multiple breast tumor markers and key molecules in microenvironment in specimens with limited available tissue and to observe qualitative and quantitative relationship between tumor cells and microenvironment dynamically and, finally, to explore the subtypes of breast cancer with different prognosis and biological characteristics. Thus, the QD-based in situ simultaneous imaging and quantitative analysis will be widely used in clinical and scientific research.

This study has some limitations. First, it was a retrospective study using a nonrandomized database, and it included only 109 breast cancer specimens, so this study needs to be validated in a large sample and prospective clinical trial. Second, the follow-up was only 5 years, so we need longer follow-up. Third, some slides were too old for QD-based in situ simultaneous imaging and quantitative detection to be performed accurately and were abandoned, so the amount of specimens was small. Fourth, collagen IV is not tumor-specific, it is a major extracellular matrix constituent, so we did not analyze its prognostic value. Nevertheless, QD-based nanotechnology provides a new method to detect multiple biomarkers. This study achieved in situ, highly sensitive, colocated molecular imaging and quantitative detection of CCL5 and collagen IV and demonstrated the prognostic value of the CCL5/collagen IV ratio in patients with luminal B (HER2-) breast cancer who received equivalent individualized treatment.

\section{Conclusion}

We capitalized on QD-based multiplex molecular imaging and computer-aided quantitative analysis technology to simultaneously and quantitatively detect CCL5 and collagen IV expression. The new indicator, CCL5/collagen IV ratio, is an effective prognostic factor in patients with luminal $B$ (HER2-) breast cancer and is more specific than CCL5. Our results demonstrate the interactions between cancer cells and microenvironment quantitatively, opening up a new field for predicting the clinical outcome in luminal B (HER2-), 
and indicate the need for more intensive investigations of the related biomarkers in this unique subtype of breast cancer.

\section{Acknowledgments}

This work was supported by funds from the Science and Technology Planning Project of Wuhu China (2017cg21 to Y-YZ), the National Natural Science Foundation of China (81201524 to 81471781 to S-RS) and the National Natural Science Foundation of China (81302314 to J-JL).

\section{Disclosure}

The authors report no conflicts of interest in this work.

\section{References}

1. DeSantis CE, Ma J, Goding Sauer A, Newman LA, Jemal A. Breast cancer statistics, 2017, racial disparity in mortality by state. CA Cancer J Clin. 2017;67(6):439-448.

2. Yanagawa M, Ikemot K, Kawauchi S, et al. Luminal A and luminal B (HER2 negative) subtypes of breast cancer consist of a mixture of tumors with different genotype. BMC Res Notes. 2012;5:376.

3. Focke CM, van Diest PJ, Decker T. St Gallen 2015 subtyping of luminal breast cancers: impact of different Ki67-based proliferation assessment methods. Breast Cancer Res Treat. 2016;159(2):1-7.

4. Effi AB, Aman NA, Koui BS, Koffi KD, Traore ZC, Kouyate M. Breast cancer molecular subtypes defined by ER/PR and HER2 status: association with clinicopathologic parameters in Ivorian patients. Asian Pac J Cancer Prev. 2016;17(4):1973-1978.

5. Holowatyj AN, Ruterbusch JJ, Ratnam M, Gorski DH, Cote ML. HER2 status and disparities in luminal breast cancers. Cancer Med. 2016; 5(8):2109-2116.

6. Bediaga NG, Beristain E, Calvo B, et al. Luminal B breast cancer subtype displays a dicotomic epigenetic pattern. Springerplus. 2016; 5(1):1-10.

7. Li ZH, Hu PH, Tu JH, Yu NS. Luminal B breast cancer: patterns of recurrence and clinical outcome. Oncotarget. 2016;7(40):65024-65033.

8. Knudsen ES, Witkiewicz AK. Defining the transcriptional and biological response to CDK4/6 inhibition in relation to ER+/HER2- breast cancer. Oncotarget. 2016;7(43):69111-69123.

9. Park C, Park K, Kim J, et al. Prognostic values of negative estrogen or progesterone receptor expression in patients with luminal B HER2negative breast cancer. World J Surg Oncol. 2016;14(1):244.

10. Rajc J, Gugić D, Fröhlich I, Marjanović K, Dumenčić B. Prognostic role of nectin-4 expression in luminal B (HER2 negative) breast cancer. Pathol Res Pract. 2017;213(9):1102-1108.

11. Cancello G, Maisonneuve P, Rotmensz N, et al. Progesterone receptor loss identifies luminal B breast cancer subgroups at higher risk of relapse. Ann Oncol. 2013;24(3):661-668.

12. Miyagawa Y, Miyake T, Yanai A, et al. Association of body mass index with risk of luminal A but not luminal B estrogen receptor-positive andHER2-negative breast cancer for postmenopausal Japanese women. Breast Cancer. 2015;22(4):399-405.

13. Criscitiello C, Disalvatore D, De Laurentiis M, et al. High Ki-67 score is indicative of a greater benefit from adjuvant chemotherapy when added to endocrine therapy in luminal B HER2 negative and node-positive breast cancer. Breast. 2014;23(1):69-75.

14. Maisonneuve P, Disalvatore D, Rotmensz N, et al. Proposed new clinicopathological surrogate definitions of luminal A and luminal B (HER2-negative) intrinsic breast cancer subtypes. Breast Cancer Res. 2014;16(3):R65.

15. Zong Y, Zhu L, Wu J, et al. Progesterone receptor status and Ki-67 index may predict early relapse in luminal B/HER2 negative breast cancer patients: a retrospective study. PLoS One. 2014;9(8):e95629.
16. Sato K, Miyashita M, Ishida T, et al. Prognostic significance of the progesterone receptor status in Ki67-high and -low luminal B-like HER2-negative breast cancers. Breast Cancer. 2016;23(2):310-317.

17. Campbell MJ, Baehner F, O'Meara T, et al. Characterizing the immune microenvironment in high-risk ductal carcinoma in situ of the breast. Breast Cancer Res Treat. 2017;161(1):17-28.

18. Cheng KK, Dickson A, Gujam FJ, McMillan DC, Edwards J. The relationship between oestrogen receptor-alpha phosphorylation and the tumour microenvironment in patients with primary operable ductal breast cancer. Histopathology. 2017;70(5):782-797.

19. Wu X, Liu H, Liu J, et al. Immunofluorescent labeling of cancer marker Her2 and other cellular targets with semiconductor quantum dots. Nat Biotechnol. 2003;21(1):41-46.

20. Chen C, Peng J, Xia H, et al. Quantum-dot-based immunofluorescent imaging of HER2 and ER provides new insights into breast cancer heterogeneity. Nanotechnology. 2010;21(9):095101.

21. Yang XQ, Chen C, Peng CW, et al. Quantum dot-based quantitative immunofluorescence detection and spectrum analysis of epidermal growth factor receptor in breast cancer tissue arrays. Int J Nanomedicine. 2011;6:2265-2273.

22. Sun JZ, Chen C, Jiang G, Tian WQ, Li Y, Sun SR. Quantum dot-based immunofluorescent imaging of Ki67 and identification of prognostic value in HER2-positive (non-luminal) breast cancer. Int JNanomedicine. 2014;9:1339-1346.

23. Peng CW, Liu XL, Chen C, et al. Patterns of cancer invasion revealed by QDs-based quantitative multiplexed imaging of tumor microenvironment. Biomaterials. 2011;32(11):2907-2917.

24. Liu XL, Peng CW, Chen C, et al. Quantum dots-based double-color imaging of HER2 positive breast cancer invasion. Biochem Biophys Res Commun. 2011;409(3):577-582.

25. Mi Z, Bhattacharya SD, Kim VM, Guo H, Talbot LJ, Kuo PC. Osteopontin promotes CCL5-mesenchymal stromal cell mesenchymal stromal cell-mediated breast cancer metastasis. Carcinogenesis. 2011; 32(4):477-487.

26. Forst B, Hansen MT, Klingelhöfer J, et al. Metastasis-inducing S100A4 and RANTES cooperate in promoting tumor progression in mice. PLoS One. 2010;5(4):e10374.

27. Rizvi SB, Rouhi S, Taniguchi S, et al. Near-infrared quantum dots for HER2 localization and imaging of cancer cells. Int J Nanomedicine. 2014;9:1323-1337.

28. Michalska M, Florczak A, Dams-Kozlowska H, Gapinski J, Jurga S, Schneider R. Peptide-functionalized ZCIS QDs as fluorescent nanoprobe for targeted HER2-positive breast cancer cells imaging. Acta Biomater. 2016;35:293-304.

29. Xu H, Chen C, Peng J, et al. Evaluation of the bioconjugation efficiency of different quantum dots as probes for immunostaining tumor-marker proteins. Appl Spectrosc. 2010;64(8):847-852.

30. Chen C, Sun SR, Gong YP, et al. Quantum dots-based molecular classification of breast cancer by quantitative spectroanalysis of hormone receptors and HER2. Biomaterials. 2011;32(30):7592-7599.

31. Velaei K, Samadi N, Barazvan B, Soleimani Rad J. Tumor microenvironment-mediated chemoresistance in breast cancer. Breast. 2016;30: 92-100.

32. Weitzenfeld P, Meshel T, Ben-Baruch A. Microenvironmental networks promote tumor heterogeneity and enrich for metastatic cancer stem-like cells in luminal-A breast tumor cells. Oncotarget. 2016;7(49):81123-81143.

33. Chen X, Wang Y, Nelson D, et al. CCL2/CCR2 regulates the tumor microenvironment in HER-2/neu-driven mammary carcinomas in mice. PLoS One. 2016;11(11):e0165595.

34. Svensson S, Abrahamsson A, Rodriguez GV, et al. CCL2 and CCL5 are novel therapeutic targets for estrogen-dependent breast cancer. Clin Cancer Res. 2015;21(16):3794-3805.

35. Yi EH, Lee CS, Lee JK, et al. STAT3-RANTES autocrine signaling is essential for tamoxifen resistance in human breast cancer cells. Mol Cancer Res. 2013;11(1):31-42.

36. Niwa Y, Akamatsu H, Niwa H, Sumi H, Ozaki Y, Abe A. Correlation of tissue and plasma RANTES levels with disease course in patients with breast or cervical cancer. Clin Cancer Res. 2001;7(2):285-289. 
37. Yaal-Hahoshen N, Shina S, Leider-Trejo L, et al. The chemokine CCL5 as a potential prognostic factor predicting disease progression in stage II breast cancer patients. Clin Cancer Res. 2006;12(15):4474-4480.

38. Bièche I, Lerebours F, Tozlu S, Espie M, Marty M, Lidereau R. Molecular profiling of inflammatory breast cancer: identification of a poorprognosis gene expression signature. Clin Cancer Res. 2004;10(20): 6789-6795.

39. Vittoria D, Domenico L, Rosaria AM, et al. Adipose microenvironment promotes triple negative breast cancer cell invasiveness and dissemination by producing CCL5. Oncotarget. 2016;7(17):24495-24509.

40. Gao D, Rahbar R, Fish EN. CCL5 activation of CCR5 regulates cell metabolism to enhance proliferation of breast cancer cells. Open Biol. 2016;6(6):160122.

41. Yasuhara R, Irié T, Suzuki K, et al. The $\beta$-catenin signaling pathway induces aggressive potential in breast cancer by up-regulating the chemokine CCL5. Exp Cell Res. 2015;338(1):22-31.

42. Zhang Q, Qin J, Zhong L, et al. CCL5-mediated Th2 immune polarization promotes metastasis in luminal breast cancer. Cancer Res. 2015; 75(20):4312-4321.

43. Reigosa A, Hardisson D, Sanzi F, et al. Characterization of patientderived tumor xenografts (PDXs) as models for estrogen receptor positive (ER+HER2 - and ER+HER2+) breast cancers. J Steroid Biochem Mol Biol. 2017;170:65-74.
44. Reigosa A, Hardisson D, Sanzi F, Caleiras E, Saldivia F, Fernández A. Subclassification of the molecular types of breast cancer based on the expression of immunohistochemical markers and evolution. Invest Clin. 2016;57(2):187-216.

45. Pendharkar N, Gajbhiye A, Taunk K, et al. Quantitative tissue proteomic investigation of invasive ductal carcinoma of breast with luminal B HER2positive and HER2 enriched subtypes towards potential diagnostic and therapeutic biomarkers. J Proteomics. 2016;132:112-130.

46. Ohlund D, Lundin C, Ardnor B, Oman M, Naredi P, Sund M. Type IV collagen is a tumour stroma-derived biomarker for pancreas cancer. Br J Cancer. 2009;101(1):91-97.

47. Cavo M, Fato M, Peñuela L, Beltrame F, Raiteri R, Scaglione S. Microenvironment complexity and matrix stiffness regulate breast cancer cell activity in a 3D in vitro model. Sci Rep. 2016;6:35367.

48. Chan N, Willis A, Kornhauser N, et al. Influencing the tumor microenvironment: phase 2 study of copper depletion with tetrathiomolybdate in high risk breast cancer and preclinical models of lung metastases. Clin Cancer Res. 2017;23(3):666-676.

49. Okabe M, Toh U, Iwakuma N, et al. Predictive factors of tumor immune microenvironment for long-term follow-up in early stage breast cancer. Cancer Sci. 2016;108(1):81-90.
International Journal of Nanomedicine

\section{Publish your work in this journal}

The International Journal of Nanomedicine is an international, peerreviewed journal focusing on the application of nanotechnology in diagnostics, therapeutics, and drug delivery systems throughout the biomedical field. This journal is indexed on PubMed Central, MedLine, CAS, SciSearch $®$, Current Contents $\AA /$ Clinical Medicine,

\section{Dovepress}

Journal Citation Reports/Science Edition, EMBase, Scopus and the Elsevier Bibliographic databases. The manuscript management system is completely online and includes a very quick and fair peer-review system, which is all easy to use. Visit http://www.dovepress.com/ testimonials.php to read real quotes from published authors. 